\title{
Giant Cell-Rich Tumors of Bone
}

\author{
Wolfgang Hartmann, $\mathrm{MD}^{\mathrm{a}}$, Dorothee Harder, $\mathrm{MD}^{\mathrm{b}}$, Daniel Baumhoer, $\mathrm{MD}^{\mathrm{c}, *}$
}

\author{
KEYWORDS \\ - Giant cell tumor • Giant cell granuloma $\bullet$ Non-ossifying fibroma $\bullet$ Aneurysmal bone cyst \\ - USP6-rearranged lesions
}

Key points

- Multinucleated giant cells can be found in a distinct group of bone tumors and generally represent bystanders of the actual neoplastic population

- Most giant cell-rich bone tumors show characteristic molecular alterations that can aid in the differential diagnosis and primarily involve mutations of the H3.3, KRAS, and FGFR1 genes as well as rearrangements of the USP6 gene

- Benign fibrous histiocytoma (BFH) is no longer listed as a separate entity in the latest WHO classification and believed to represent giant cell tumor with regressive changes

- Giant cell lesion of the small tubular bones has also been eliminated as a distinct entity in the latest WHO classification and thought to represent solid aneurysmal bone cyst

- Rearrangements of the USP6 gene are not limited to classical aneurysmal bone cysts but occur in a variety of formerly "difficult to classify" lesions

\section{ABSTRACT}

$\mathbf{T}$ he term giant cell-rich tumors of bone refers to a shared morphologic pattern in a group of different osseous lesions, that is, the abundance of osteoclastlike giant cells. Fitting with a broad spectrum of clinical presentations and biological behavior, the recent detection of characteristic molecular alterations in giant cell tumor of bone (H3-3), nonossifying fibroma (KRAS, FGFR1), giant cell granuloma of the jaws (KRAS, FGFR1, TRPV4), and aneurysmal bone cyst (USP6) have contributed significantly to the biological understanding of these morphologically related but clinically distinct lesions and their systematic classification, highlighting differences and pathogenic relationships.

\section{GIANT CELL TUMOR OF BONE}

\section{INTRODUCTION}

Conventional giant cell tumor constitutes $4 \%$ to $5 \%$ of all primary bone tumors. This tumor is considered a locally aggressive and rarely metastasizing neoplasm that can undergo malignant transformation into a high-grade sarcoma. ${ }^{1}$ Giant cell tumor of bone (GCT) predominantly develops in the epiphyseal/metaphyseal region of long tubular bones after skeletal maturity ( $>20$ years). In less than $10 \%$ of cases, tumors occur while the growth plates are still open and are then centered more proximally in the metaphyses. ${ }^{2}$ Most commonly involved sites include the distal femur, the proximal tibia, and the distal radius. In

\footnotetext{
a Division of Translational Pathology, Gerhard-Domagk-Institut of Pathology, University Hospital Münster, Albert-Schweitzer-Campus 1, Münster 48149, Germany; ${ }^{b}$ Department of Radiology, University Hospital Basel, University of Basel, Petersgraben 4, Basel 4031, Switzerland; ' ${ }^{\circ}$ Bone Tumor Reference Center, Institute of Medical Genetics and Pathology, University Hospital Basel, University of Basel, Schoenbeinstrasse 40, Basel 4031, Switzerland

* Corresponding author.

E-mail address: daniel.baumhoer@usb.ch
} 
the spine, the vertebral bodies are affected. GCT is exceptionally rare in the craniofacial bones.

\section{IMAGING FEATURES}

As GCTs lack formation of mineralized matrix, they appear purely lytic in radiographs and computed tomographic (CT) scans without sclerotic margins. GCTs are usually large, intramedullary, eccentrically located, and well defined (Fig. 1A). Cortical scalloping and penetration can occur in advanced lesions, but aggressive periosteal reactions are usually absent. MRI of GCT shows nonspecific, usually heterogeneous signal intensity on T1weighted images before and after contrast administration; T2-weighted images are also nonspecific but can demonstrate (pseudo-)cystic change (fluid-fluid levels, see Fig. 1C).

\section{GROSS AND MICROSCOPIC FEATURES}

Tumors are mostly solid and consist of friable brown tissue with varying amounts of hemorrhage, cystic change, and necrosis. Tumors usually measure 5 to $15 \mathrm{~cm}$ in diameter.

Microscopically, abundant and remarkably large multinucleated giant cells are the hallmark of the disease. Although intermingled smaller giant cells can occur in a variety of different lesions, the amount and size of giant cells demonstrating more than 50 nuclei per tissue section is typical for GCT (Fig. 1B). The neoplastic cell population, however, is composed of mostly monomorphic oval or polygonal cells arranged in a haphazard manner; it shows ill-defined cell borders and a slightly eosinophilic cytoplasm. Nuclei tend to appear round to ovoid with vesicular chromatin and central nucleoli; they are practically identical to the nuclei of the giant cell component. Mitotic activity, mild to moderate cytologic atypia, and areas of necrosis can be observed, as well as vascular invasion. GCT can undergo regressive changes with prominent accumulation of siderophages and foam cells; other secondary changes include (pseudo-)cystic change and reactive new bone formation.

Following antiresorptive therapy (eg, with denosumab), the microscopic appearance markedly changes. The multinucleated giant cells disappear, and the lesional mononuclear cells decrease and partly transform into osteoblasts forming varying amounts of immature woven bone trabeculae. ${ }^{3,4}$ This observation is in line with gene expression profiles of the mononuclear component of GCT that

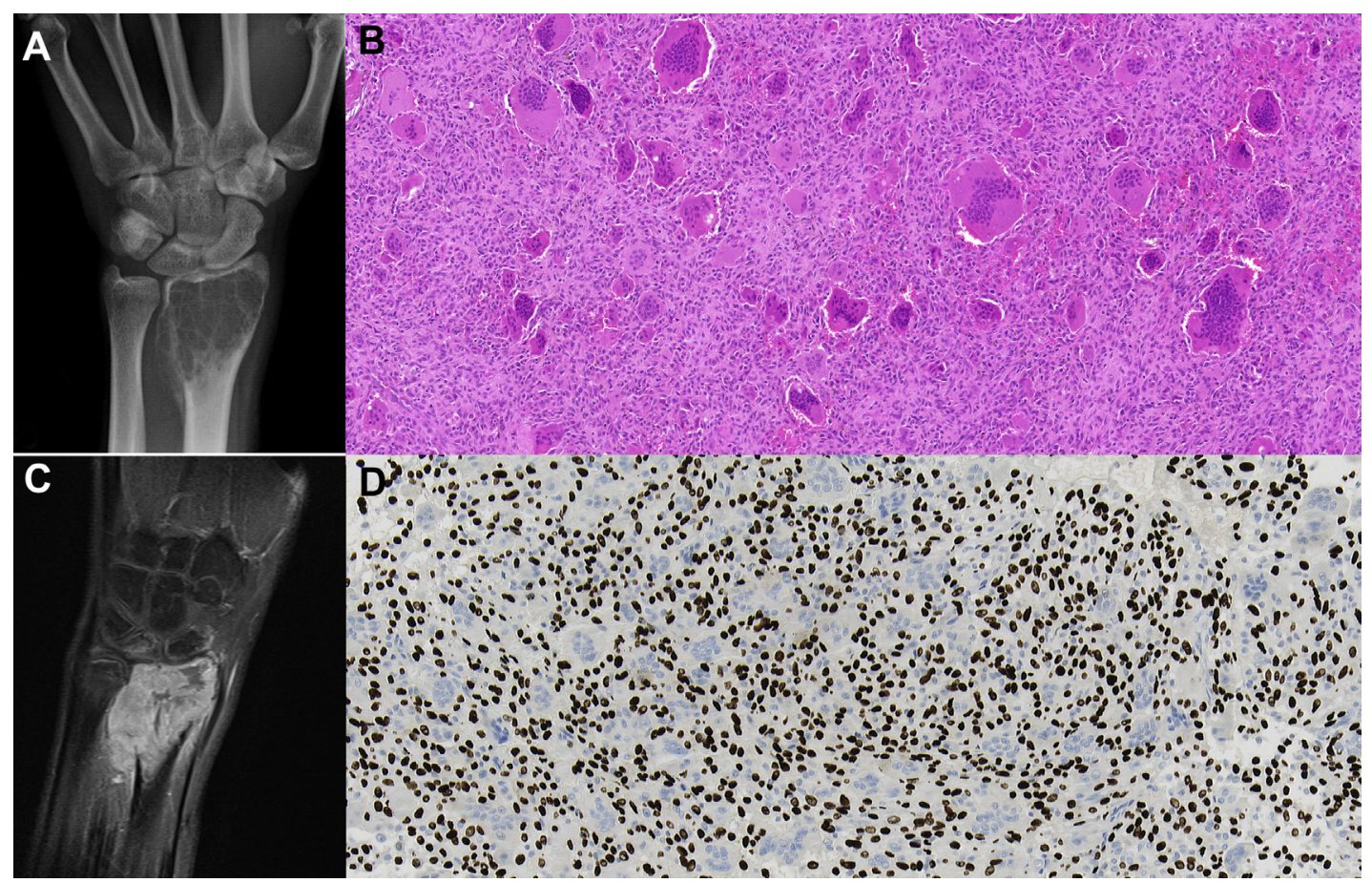

Fig. 1. GCT in the distal radius of a 17-year old boy. The conventional radiograph shows a well-demarcated, slightly expansile lytic lesion extending to the subchondral bone $(A)$ with heterogeneous enhancement in a T1-weighted, fat-saturated MRI sequence after contrast administration (C). Microscopically, multiple (nonneoplastic) large giant cells are embedded in a background of monomorphic mononuclear spindle cells arranged in a haphazard manner $(B)$ [hematoxylin-eosin, original magnification, 50x]. H3.3 G34W immunoreactivity can be observed in the mononuclear spindle cells $(D)$ [original magnification, 100x]. 
resemble those of cells with early osteoblastic differentiation. ${ }^{5}$ In some cases, reactive atypia can be striking and mimic malignant transformation. ${ }^{3}$

Malignant GCT can show different kinds of presentation. Typically, parts of a regular GCT can be found adjacent to a high-grade sarcoma, similar to dedifferentiated liposarcoma or chondrosarcoma. The characteristic histone H3.3 mutation of GCT usually remains detectable in the sarcomatous component, and malignant transformation can occur in the primary manifestation or after local recurrences of the GCT. Osteosarcoma (OS) or undifferentiated pleomorphic sarcoma (UPS) comprise the most common histologic patterns found in malignant GCT. OS or UPS without a detectable GCT component but revealing an H3.3 mutation most likely also represent malignant GCT. Genetically, malignant GCT resembles OS and UPS and shows a high degree of aneuploidy. ${ }^{6}$

\section{IMMUNOPHENOTYPE AND MOLECULAR PATHOLOGY FEATURES}

Virtually all GCTs (96\%) harbor mutations in the H3-3A gene specifically involving Glycine 34, with G34W (p.Gly34Trp) accounting for $90 \%$ of cases and G34L (p.Gly34Lys) for a smaller minority..$^{7-9}$ The G34W mutation can be detected reliably by immunohistochemistry showing nuclear positivity in the mononuclear cell population only (Fig. 1D). The functional impact is still largely unknown so far, but recent studies point to a massive effect on the global epigenetic landscape that seems to impair normal osteogenic differentiation. ${ }^{10}$ Apart from this driver mutation, GCTs show a relatively low mutation burden and a flat copy number profile.

\section{DIFFERENTIAL DIAGNOSIS}

The histology in the typical clinical and imaging context is fairly diagnostic for GCT without additional analyses, whereas H3.3 testing confirmed that giant cell granulomas of the jaws, aneurysmal bone cyst $(A B C)$, giant cell lesions of the small tubular bone, and non-ossifying fibroma are not related to GCT. On the other hand, lesions formerly classified as $B F H$, histologically resembling non-ossifying fibroma but mainly occurring later in life and centered in the epiphyses of long tubular bones, turned out to represent giant cell tumors with marked regressive changes and are no longer listed as a separate entity in the current World Health Organization (WHO) classification (Fig. 2). In case of doubt, immunohistochemical testing for the G34W mutation is usually sufficient to confirm GCT. The detection of rarer mutations still requires sequencing. ${ }^{11}$

\section{SUMMARY AND PROGNOSIS}

Conventional GCT recurs locally following curettage in $15 \%$ to $50 \%$, usually within 2 years. Tumors can occasionally metastasize to the lungs, which is believed to result from embolization of intravascular growth. Metastases occur in 3\% to $7 \%$ of cases, often following local recurrence; show limited growth dynamics; and can regress spontaneously. The prognosis of malignant GCT is similar to those of other high-grade sarcomas of bone.

\section{CLINICS CARE POINTS}

- Most cases present as lytic mass lesions in the epiphyseal region of long tubular bone in patients older than 20 years

- Abundant large multinucleated giant cells distributed evenly among mononuclear spindle cells are characteristic; H3.3 testing can confirm the diagnosis

- Regressive changes, either following denosumab or without prior treatment (formerly known as BFH), can significantly alter the histologic and radiologic appearance of GCT

- Metastases can occur but often follow an indolent clinical course; primary/secondary malignant GCTs represent high-grade bone sarcomas requiring a multidisciplinary treatment approach

\section{NON-OSSIFYING FIBROMA OF BONE (NOF)}

\section{INTRODUCTION}

NOF is the most common benign tumor of bone. ${ }^{12}$ As it usually follows an asymptomatic course and undergoes spontaneous resolution, most cases are clinically not recognized, which is why the actual incidence of NOF is difficult to determine. It has been estimated, however, that $30 \%$ to $40 \%$ of children develop one or even multiple occult lesions during skeletal growth. ${ }^{13}$ The first edition of the WHO classification of bone tumors published in 1972 described NOF as a nonneoplastic bone lesion of "obscure etiology"; the second edition from 1993 continued to recognize NOF as a tumorlike lesion, probably representing a developmental anomaly. ${ }^{14,15}$ In 2002 the third edition of the WHO classification was published and did not list NOF as a separate bone tumor entity; in the fourth edition from 2013 NOF was described together with benign fibrous histiocytoma as a fibrohistiocytic lesion. ${ }^{16}$ After recurrent pathogenic 

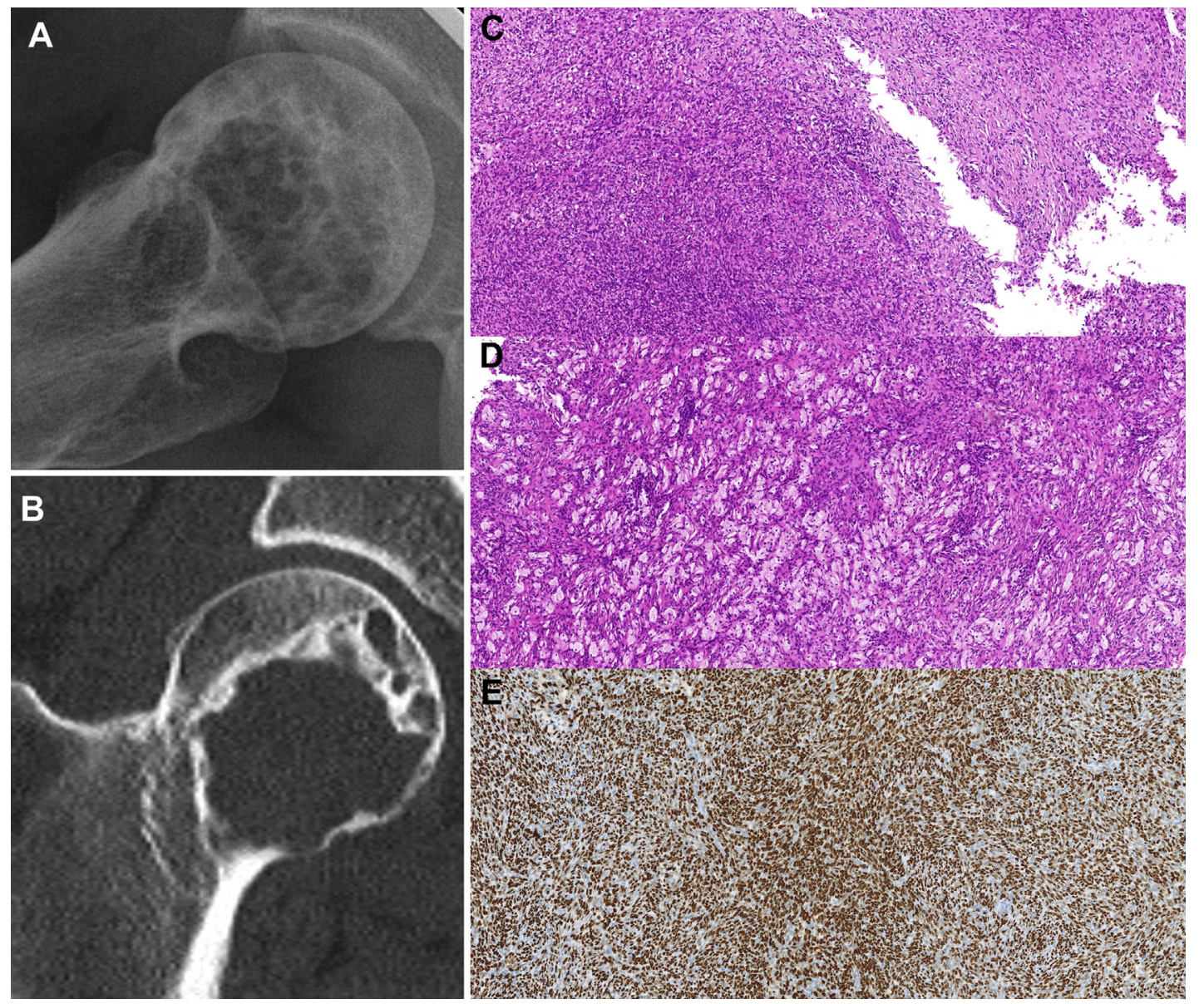

Fig. 2. GCT with regressive changes (former BFH). Conventional radiographs $(A)$ and coronal CT reconstruction ( $B$ ) of the proximal femur of a 33-year old woman show a well-demarcated osteolysis of the femoral head with a peripheral sclerotic rim. Microscopically, a dense spindle cell proliferation with only few intermingled multinucleated giant cells $(C)$ and extensive foam cell change [hematoxylin-eosin, original magnification, 50x] $(D)$ is visible, resembling non-ossifying fibroma (NOF) of bone [hematoxylin-eosin, original magnification, 50x]. $(E)$ The tumor cells are consistently positive for H3-3A (G34W mutation 50x).

mutations in the mitogen-activated protein (MAP) kinase signaling pathway were identified recently, NOF was finally recognized as a benign neoplasm and is now listed under the osteoclastic giant cellrich tumors in the current and fifth edition of the WHO blue book.

NOF exclusively develops in the first 2 decades of life and generally affects the metaphyses of long tubular bones, mainly around the knee and in the distal tibia. Multiple lesions can occur sporadically and also in distinct syndromes, mostly belonging to the spectrum of RASopathies and including neurofibromatosis type 1 (NF1) and JaffeCampanacci syndrome.

\section{IMAGING FEATURES}

The presentation of NOF on plain radiographs is usually so characteristic that no biopsy confirmation is required. Lesions appear well defined, polylobulated (grapelike), and lytic. Lesions are centered in the cortex and show sclerotic and scalloped borders (Fig. 3A, B). Remarkably, (residual) NOF is only rarely encountered in adults, which is probably caused by spontaneous regression and resolution over time.

\section{GROSS AND MICROSCOPIC FEATURES}

Most NOFs consist of solid brown tissue with variable amounts of hemorrhage and (pseudo-) cystic change. Necrosis is usually only present after a prior pathologic fracture.

Histologically, the tumors are composed of histiocytelike spindle cells arranged in storiform patterns. Mitoses can be found but are usually scant. There is no significant cytologic atypia. Throughout the lesion, intermingled 


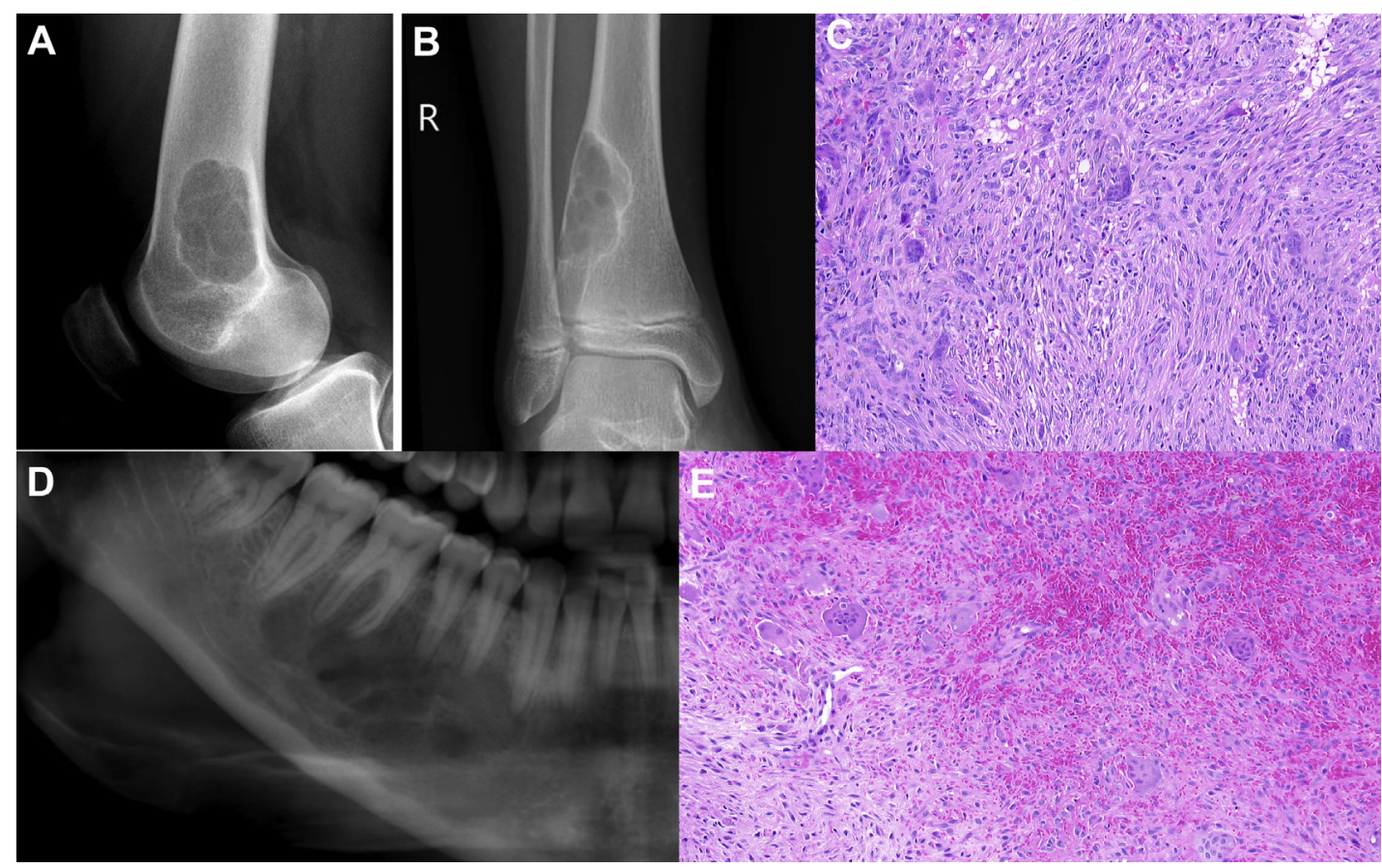

Fig. 3. Plain radiographs of non-ossifying fibroma in the distal femur of a 16 -year old girl $(A)$ and in the distal tibia of a 14-year old boy $(B)$ displaying well-defined, eccentric, and lobulated radiolucent lesions with sclerotic borders. Microscopically, histiocytelike spindle cells are arranged in storiform patterns with intermingled osteoclastlike giant cells $(C)$ [hematoxylin-eosin, original magnification, 50x]. (D) Mandibular central giant cell granuloma in a 28-year old woman presenting as a well-circumscribed lytic lesion. Microscopically, monomorphic mononuclear spindle cells and scattered multinucleated giant cells can be observed in association with areas of hemorrhage $(E)$ [hematoxylin-eosin, original magnification, 50x].

multinucleated giant cells can be observed that appear rather small (>10 nuclei per tissue section) and cluster around areas of hemorrhage (Fig. 3C). Aggregations of siderophages and foam cells are other typical findings, the background can be rich in collagen fibers. Reactive new bone formation is rare (non-ossifying fibroma) but can be seen following a fracture. The histology of NOF strongly resembles that of giant cell granuloma of the jaws.

\section{IMMUNOPHENOTYPE AND MOLECULAR PATHOLOGY FEATURES}

In the vast majority of cases, NOF can be reliably diagnosed without additional analysis but requires radiologic correlation. The immunophenotype is nonspecific and highlights the histiocytic differentiation of the lesional cells. Sequencing revealed mutually exclusive hotspot mutations affecting KRAS and FGFR1 in greater than $80 \%$ of cases, which strongly argues for a neoplastic nature. ${ }^{11}$ The reason why NOF ceases to grow at some point and completely resolves over time without leaving a trace remains unclear. A similar mechanism like in fibrous dysplasia in which the lesional cells carrying the GNAS mutation get diminished over time by undergoing apoptosis might be a possible explanation. ${ }^{17}$

\section{DIFFERENTIAL DIAGNOSIS}

In the appropriate clinical and imaging context, the diagnosis of NOF is usually straightforward. A nearly identical morphology in the jaws can be found in giant cell granuloma (GCG), which is outlined in more detail later discussion. Solid parts of $A B C$ can appear similar microscopically but lack the typical radiologic appearance of NOF. In case of doubt, a fluorescence in situ hybridization (FISH) analysis of the USP6 gene is helpful to support the diagnosis of ABC. Another histologic mimic is GCT with regressive changes, which was formerly believed to represent a distinct lesion designated as benign fibrous histiocytoma (BFH). The histology can appear identical to NOF, but age and the involved sites mirror the distribution of GCT. Immunohistochemical detection of the H3.3 mutation is an easy way to diagnose or rule out GCT with regressive changes. 


\section{SUMMARY AND PROGNOSIS}

Surgical pathologists only infrequently encounter NOF in their daily routine because it is only biopsied or curetted in case of (imminent) fracture. The prognosis is excellent, and local recurrences are rare. Malignant transformation has not been described so far. As the KRAS and FGFR1 mutations found in NOF are virtually absent in conventional OS, a relationship between these 2 lesions seems highly unlikely.

\section{CLINICS CARE POINTS}

- Most common benign tumor of bone

- Pathognomonic presentation on plain radiographs showing a well-defined, grapelike osteolysis with a sclerotic rim centered in the cortex of the metaphysis of long tubular bones

- Mutations in the MAP kinase signaling pathway drive NOF

- Histologic mimics can be easily ruled out by radiologic correlation and few molecular tests (H3.3, USP6)

\section{GIANT CELL GRANULOMA OF THE JAWS (GCG)}

\section{INTRODUCTION}

Giant cell granuloma is a benign and sometimes locally aggressive tumor of the jaws; it can develop inside the bone (central giant cell granuloma of the jaws [GCG]) or in the gingival/alveolar mucosa (peripheral GCG). Central GCG most commonly affects patients in the first 2 decades of life and accounts for $10 \%$ of all benign tumors of the jaws. The anterior aspects of the mandible (and maxilla) are most frequently involved. ${ }^{18}$ Peripheral GCG (synonym giant cell epulis) usually occurs later in life (fifth and sixth decades) and most commonly develops in the mandible. ${ }^{19}$

GCG has long been considered to be of reactive nature, and central GCG has also been referred to as reparative GCG. In the current WHO classification of head and neck tumors published in early 2017 , central GCG is defined as a benign "lesion" and peripheral GCG is described to result from chronic irritation. In 2018, Gomes and colleagues, ${ }^{20}$ however, discovered recurrent mutations in the MAP kinase signaling pathway in both central and peripheral GCG, strongly arguing in favor of a neoplastic origin.

Imaging Features

Central GCG typically presents as a slowly growing, well-delineated, and expansile osteolysis with cortical thinning (Fig. 3D). If large, a multilobulated and/or soap bubble appearance can be observed. Locally aggressive lesions show cortical penetration and soft tissue infiltration. Tooth resorption or displacements are sometimes evident. Peripheral GCG can erode the underlying bone, which can be visible on radiographs or sectional images.

\section{GROSS AND MICROSCOPIC FEATURES}

GCGs have a similar morphology independent of their site of origin. The tissue is brittle and brown with varying amounts of hemorrhage and (pseudo-)cystic change.

Microscopically, GCGs consist of monomorphic and histiocytelike spindle cells arranged in haphazard lobules. Multinucleated giant cells are usually abundant and unevenly distributed throughout the tumor. These cells are rather small ( $<10$ nuclei per tissue section) and cluster around areas of hemorrhage (Fig. 3E). The tumor is incompletely subdivided by fibrous septa containing woven bone trabeculae. Secondary changes include accumulation of siderophages, and a thin peripheral rim of reactive new bone formation may be present. Mitotic activity can vary but is usually low. Significant cytologic atypia is not a typical feature of GCG. With the exception of the fibrous septa, the histology is very similar to non-ossifying fibroma (NOF) of the peripheral skeleton.

\section{IMMUNOPHENOTYPE AND MOLECULAR PATHOLOGY FEATURES}

Immunohistochemistry is unspecific and not useful. The mononuclear cells express histiocytic markers including CD68 and CD163. Sequencing analyses have revealed recurrent KRAS and FGFR1 mutations, similar to non-ossifying fibroma of bone, in both central and peripheral GCG. In addition, TRPV4 mutations have been exclusively detected in central GCG (and neither in peripheral GCG nor in NOF so far). ${ }^{20}$ This finding is in line with the fact that GCGs can develop in several syndromes, all belonging to the spectrum of RASopathies, including NF1 as well as Noonan, LEOPARD, and Jaffe-Campanacci syndromes. Notably, some patients develop both GCG and NOF. Multiple GCGs point to a syndrome-related pathogenesis.

\section{DIFFERENTIAL DIAGNOSIS}

Brown tumors caused by hyperparathyroidism are rare in the Western population but can appear histologically identical to GCGs.

Syndrome-related GCGs are also virtually indistinguishable from sporadic cases and can be the initial presenting symptom. Even in the absence of more specific signs of Noonan syndrome or 
NF1, a thorough clinical examination or screening for the relevant germline alterations should be considered, particularly in patients with multiple GCGs. Histologically, some cases show a more prominent spindle cell component with lesser giant cells and a notably storiform pattern of growth without being specific. Lesions tend to reach a considerable size and behave locally aggressive. ${ }^{21}$

Cherubism is a rare benign disease characterized by symmetric enlargement of all 4 quadrants of the jaws. Cherubism is caused by a mutation of the SH3BP2 gene with approximately $50 \%$ being inherited in an autosomal dominant trait and $50 \%$ arising as de novo mutations. The disease manifests before the age of 6 years and is usually easy to diagnose in the appropriate clinical and imaging contexts. Histologically, however, cherubism appears similar to (syndrome-related) GCG.

$A B C$ is generally characterized by a multicystic architecture composed of blood-filled spaces subdivided by fibrous septa containing multinucleated giant cells. Imaging shows expansile lesions with fluid-fluid levels in MRI. Some ABCs, however, lack the defining cystic appearance and strongly resemble GCGs microscopically; they are referred to as solid ABCs. If this differential diagnosis is considered, USP6 FISH analysis might be helpful to substantiate it.

Conventional GCT practically does not exist in the jaws. Histologically, it shows a higher number and more evenly distributed giant cells that contain more than 50 nuclei per tissue section. H3-3A mutations are absent in GCG.

\section{SUMMARY AND PROGNOSIS}

Peripheral GCGs are usually excised and recur only infrequently, particularly if the underlying periosteum is included in the excision. Central GCGs are frequently treated by curettage. Recurrence rates differ depending on the aggressiveness of individual lesions.

\section{CLINICS CARE POINTS}

- Benign tumors exclusively occurring in the craniofacial skeleton and can be clinically classified as nonaggressive and locally aggressive types

- Sporadic cases are usually solitary and commonly affect the anterior mandible; multiple lesions point to a syndrome-related cause

- Mutations in the MAP kinase signaling cascade drive GCG (KRAS, FGFR1, TRPV4)

- Histologic mimics can be ruled out by thorough clinical/imaging correlation and laboratory tests (parathormone). Molecular tests are rarely needed (USP6 FISH)

\section{ANEURYSMAL BONE CYST}

\section{INTRODUCTION}

$A B C$ is another lesion of bone that has originally been assumed to be of reactive nature. In the first 2 editions of the WHO classification, it was listed under the tumorlike lesions; in the third and fourth editions it was listed under the category of tumors of undefined neoplastic nature. The first evidence of underlying genetic rearrangements involving the USP6 gene date back to 1991 and have been described in the WHO blue book since its third edition. ${ }^{22}$ However, it is only since the fourth edition published in 2013 that $A B C$ has been defined as a true neoplasm and regarded to be of intermediate biological potential (locally aggressive). In the current and fifth edition, $A B C$ is listed under the osteoclastic giant cell-rich tumors; disputably the editors have revised the biological behavior from locally aggressive to benign. ${ }^{23}$

$\mathrm{ABC}$ usually affects children and adolescents; $80 \%$ of cases occur in the first 2 decades of life. Typically, the metaphyses of long tubular bones and the posterior elements of the vertebrae are involved; patients present with pain and swellings.

\section{IMAGING FEATURES}

Radiologically, ABCs appear expansile, lytic, and eccentric with well-defined margins and commonly a thin shell of reactive bone. Highly aggressive features and periosteal reactions are not typical for $A B C$, but ill-defined margins, penetration of the cortex, and an extraosseous extension are also possible. MRI highlights fluid-fluid levels that are highly characteristic (Fig. 4A); contrast administration primarily shows septal enhancement. Solid components can be present but are usually not predominating and should raise suspicion of other differential diagnoses, including telangiectatic osteosarcoma (TAEOS).

\section{GROSS AND MICROSCOPIC FEATURES}

The multicystic architecture of $A B C$ shows bloodfilled spaces of varying size that are separated by thin, tan-white septa. Solid areas appear brown and fragile.

Microscopically, the cyst walls are composed of monomorphic and fibroblastlike spindle cells and scattered multinucleated giant cells. Mitotic activity can be brisk, but high-grade atypia and atypical mitoses do not occur in ABC. Both giant cells and mononuclear spindle cells form smooth and 


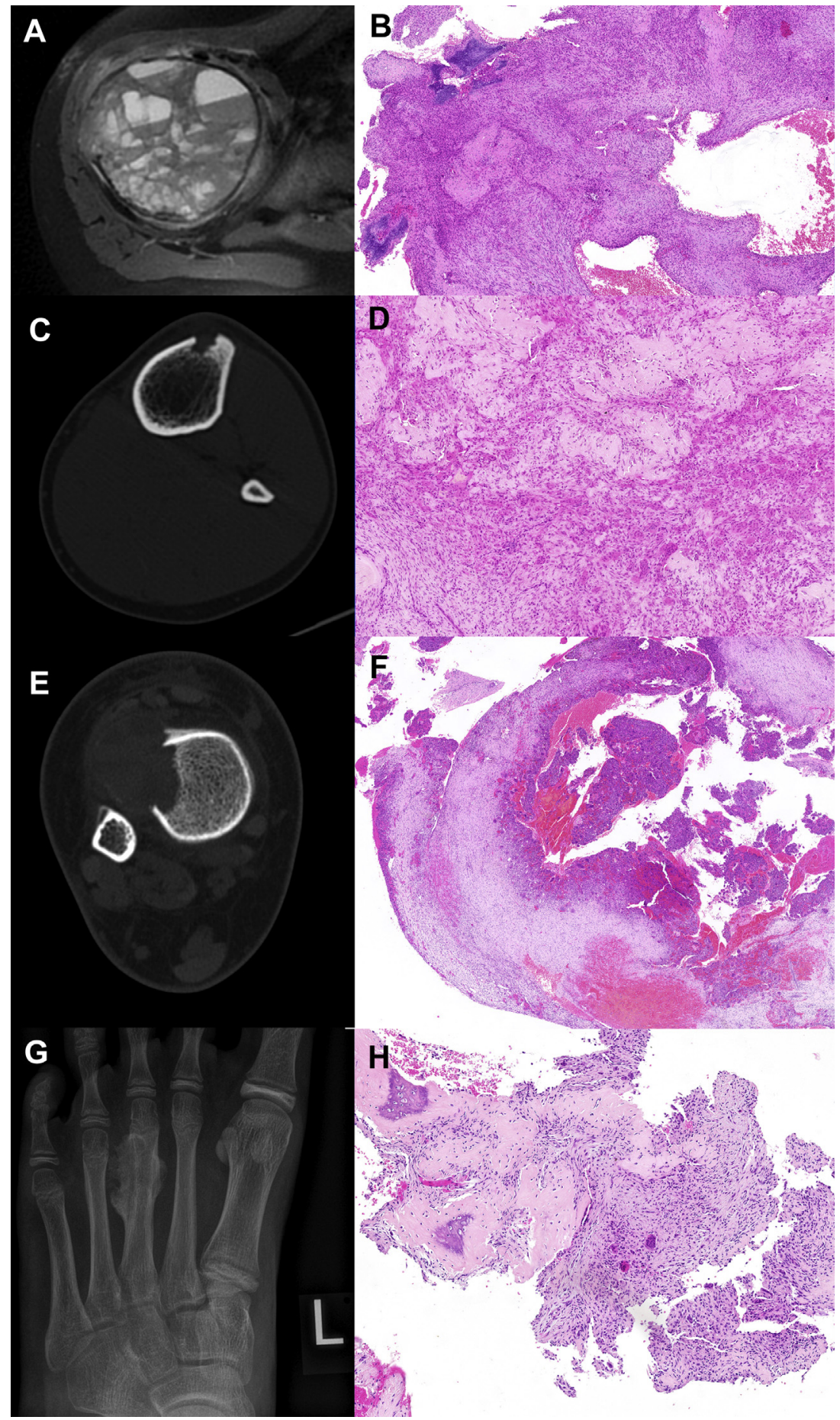

Fig. 4. Different tumors with USP6 rearrangement. Conventional $A B C$ of the right proximal humerus of a 10-year-old boy showing multiple fluid-fluid levels on T2weighted MRI $(A)$. Histologically, solid and (pseudo-)cystic areas can be distinguished. The lesion contains an immature and reactive new bone formation that appears partly basophilic (so-called blue bone) (B) [hematoxylin-eosin, original magnification, 25x]. CT scans of intracortical $A B C$ in the left tibia of a 19-year-old man (C) and in the right tibia of a 13-year old girl (E) appear lytic and locally aggressive but show a typical morphology of partly solid $A B C(D, F)$ [hematoxylin-eosin, original magnification, 50x]. Juxtacortical $A B C$ in the third metatarsal bone of a 13-year old boy presenting with a broad ossification (G, radiograph). Histologically, only minor parts of the lesion resemble $A B C$ adjacent to abundant and reactive new bone formation $(H)$ [hematoxylin-eosin, magnification, 50x]. continuous flattened layers that line the (pseudo-) cystic spaces filled by blood and serous fluid (Fig. 4B). Siderophages can be numerous, and reactive new bone formation is another common finding. In at least a third of cases, the woven bone is mineralized in an irregular fashion and appears bluish (so-called blue bone, see Fig. 4B). This peculiar matrix is not specific but a highly characteristic feature of $A B C$. Necrosis is uncommon if not secondary to pathologic fracture. Solid components show a similar cellular composition. 


\section{IMMUNOPHENOTYPE AND MOLECULAR PATHOLOGY FEATURES}

Immunohistochemistry of $A B C$ is nonspecific and generally not helpful in the diagnosis. Approximately $70 \%$ of $A B C$ s show rearrangements of the USP6 gene that fuses with various partner genes including $\mathrm{CDH} 11$ (most common, $30 \%$ of cases), THRAP3, CNBP, OMD, COL1A1, CTNNB1, STAT3, FOSL2, EIF1, SPARC, $P A F A H 1 B 1$, USP9X, and RUNX2. ${ }^{23}$ The translocations result in upregulation of USP6 transcription and are only present in the mononuclear spindle cell component. So far individual rearrangements have not been associated with a distinct clinical behavior. Notably, USP6 rearrangements have not been reported in malignant tumors so far.

\section{DIFFERENTIAL DIAGNOSIS}

Various soft tissue, bone, and gnathic tumors can show secondary changes that mimic ABC. This phenomenon has formerly been referred to as secondary $A B C$ and is not associated with USP6 rearrangements. As it only represents reactive and nonspecific changes and not a separate entity or a collision tumor, the WHO recommends using the term $A B C$-like changes.

TAEOS is an important differential diagnosis that can mimic ABC. However, in TAEOS there is frank high-grade atypia with pleomorphic high-grade nuclei and usually abundant atypical mitoses. Imaging usually shows more aggressive features and a greater amount of solid areas, but there is an overlap in imaging features with those of ABC. TAEOS does not show USP6 rearrangements.

In the jaws, solid $A B C$ can virtually be indistinguishable from GCG. However, $A B C$ is rather rare in the jaws and usually shows the classic (pseudo-)cystic presentation. Testing for USP6 rearrangements can help in ambiguous cases.

In the small tubular bones, lesions with a similar histology like GCGs have formerly been classified as giant cell lesions of the small bones. ${ }^{24}$ As most of those tumors were shown to harbor USP6 rearrangements they are no longer listed in the current WHO classification as a separate entity but considered to constitute solid $\mathrm{ABC}^{25}$ (Fig. 5).

\section{SUMMARY AND PROGNOSIS}

$A B C$ is a benign bone tumor usually occurring in children and adolescents. Recurrence rates vary significantly in the literature but probably range between $15 \%$ and $30 \%$. Malignant transformation has been described but most likely represented unusual TAEOS; so far, no convincing case of malignant transformation and underlying USP6 rearrangement has been reported.

\section{CLINICS CARE POINTS}

- Benign, expansile, and eccentrically located bone tumor developing in the metaphyses of long tubular bones and the posterior elements of the vertebrae

- Usually well defined and lytic on imaging; can show an (incomplete) sclerotic rim; aggressive imaging features are not typical, but possible; primarily cystic appearance on MRI with characteristic fluid-fluid levels

- USP6 rearrangements in $70 \%$ of cases

\section{OTHER TUMORS OF BONE WITH USP6 \\ REARRANGEMENTS}

\section{INTRODUCTION}

With increasing molecular testing more and more soft tissue and bone lesions that have formerly been regarded as reactive pseudotumors or tumorlike lesions were shown to harbor recurrent genetic alterations and are now considered true neoplasms. Highly specific findings such as $H 3.3$ mutations in GCT and chondroblastoma can be extremely helpful in routine diagnostics and often warrant a reliable diagnosis even in smaller core needle biopsies. Other genes are involved in a variety of neoplasms so a mutation or rearrangement itself limits the spectrum of differential diagnoses but is not specific for a distinct lesion per se. Remarkably, several mutations that have been considered the driver genes of highly malignant tumors have recently been shown to also drive benign and sometimes even self-limiting lesions including pathogenic KRAS hotspot mutations in non-ossifying fibroma (NOF) and EWSR1 rearrangements in simple bone cyst. ${ }^{11,26}$

USP6 rearrangements are one prominent example of highly promiscuous alterations occurring in several but so far unequivocally benign neoplasms. The consecutive discovery in distinct lesions caused confusion in the nomenclature prompting the question if a shared molecular alteration is sufficient to assume a direct relation between lesions that otherwise appear distinct from each other. As the first cases of myositis ossificans (MO) were reported to harbor USP6 rearrangements, those lesions were temporarily suggested to represent soft tissue variants of $A B C$, although clinical and microscopic features were distinct from ABC. In the meantime, it has been increasingly accepted that $\mathrm{MO}$ as well as fibro-osseous pseudotumor of digits both belong to the 

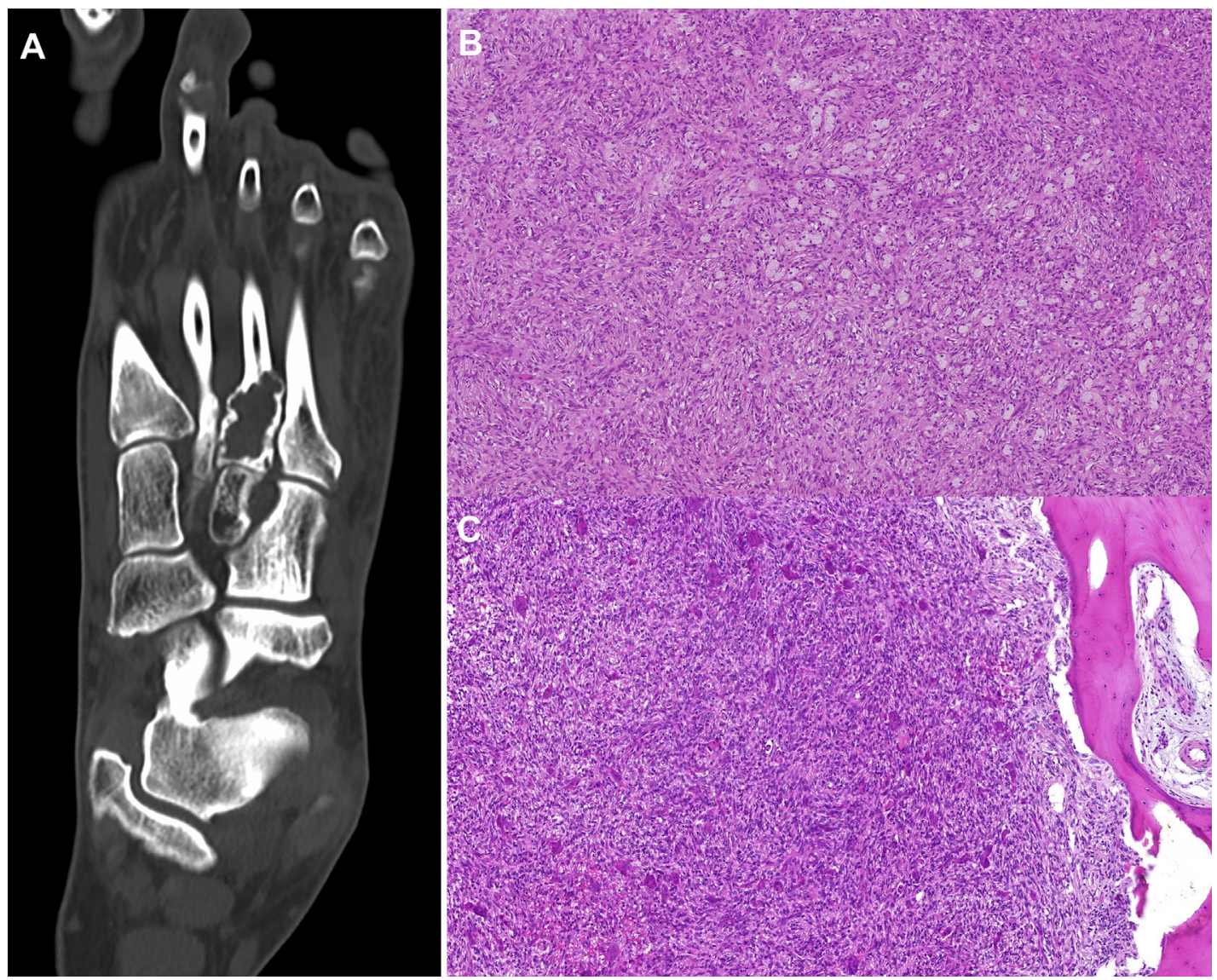

Fig. 5. Solid ABC (former giant cell lesion of the small bones). CT shows a well-defined and expansile lytic lesion in the third right metatarsal of a 29-year-old woman $(A)$. Microscopically, the tumor presents uniformly solid with foam cell changes $(B)$ [hematoxylin-eosin, original magnification, 50x] and areas resembling giant cell granuloma of the jaws $(C)$ [hematoxylin-eosin, original magnification, 50x].

spectrum of USP6-related neoplasms. ${ }^{27,28}$ The term solid $A B C$ is somehow contradictory in itself (as is giant cell tumor without giant cells) but now introduced in the WHO classification to designate lesions in the small tubular bones formerly classified as a separate entity (giant cell lesions of the small bones). ABC can also develop inside and on the surface of cortical bone, which both can appear highly unusual on imaging. USP6rearranged juxtacortical lesions can cause massive new bone formation and have been designated as periostitis ossificans, periosteal fasciitis, juxtacortical $A B C$, and cranial fasciitis, all with markedly distinct clinical presentation and behavior. $^{29,30}$

\section{IMAGING FEATURES}

Intracortical ABCs are usually lytic and well defined but can extend into the adjacent soft tissues (Fig. 4C, E). Periosteal reactions sometimes appear aggressive, indicating rapid growth; still, tumors usually present thin shells of reactive woven bone at their periphery. Solid tumors often reveal homogeneous contrast enhancement prompting a broad spectrum of differential diagnoses. The presentation of lesions developing on the surface of tubular bones varies but can be characterized by abundant new bone formation (Fig. 4G, H).

\section{GROSS, MICROSCOPIC, AND MOLECULAR PATHOLOGY FEATURES}

The macroscopic and microscopic aspects of USP6-related lesions are mostly similar to $A B C$ or nodular fasciitis. However, secondary changes can predominate and obscure the more characteristic findings. Searching for an USP6 rearrangement by FISH or RNA sequencing should be considered in unusual intracortical and juxtacortical bone tumors that at least have some resemblance to conventional $A B C$ or nodular fasciitis (Fig. 4). 


\section{SUMMARY AND PROGNOSIS}

The spectrum of USP6-related tumors is increasing and includes rare manifestations at unusual sites. If any peculiar lesion of bone with an underlying USP6 fusion transcript should be classified as (intracortical, juxtacortical, or solid) ABC will need to be decided as soon as larger series become available and potentially highlight differences in presentation and clinical behavior; currently, only few case reports have been published. In any case, the identification of USP6 rearrangements appears particularly important because, at least so far, they do not seem to occur in malignant tumors.

\section{CLINICS CARE POINTS}

- USP6-related neoplasms can show unusual presentation in bone and include solid, intracortical, and surface-related manifestations

- USP6 rearrangements have not been described in malignant tumors so far

- USP6 testing is advocated in any lesion of bone that shows at least some morphologic similarity to conventional $\mathrm{ABC}$ or nodular fasciitis

\section{ACKNOWLEDGEMENTS}

Dr DB was supported by the Swiss National Science Foundation, the Foundation of the Bone Tumor Reference Center, the Gertrude von Meissner Stiftung and the Stiftung für krebskranke Kinder, Regio Basiliensis.

\section{DISCLOSURE}

The authors have nothing to disclose.

\section{REFERENCES}

1. Flanagan AM, Larousserie F, O'Donnell PG, et al. Giant cell tumour of bone. In: Board WCoTE, editor. WHO classification of tumours, soft tissue and bone tumours. 5th edition. France: IARC Press; 2020. p. 440-6.

2. Al-Ibraheemi A, Inwards CY, Zreik RT, et al. Histologic spectrum of giant cell tumor (GCT) of bone in patients 18 years of age and below: a study of 63 patients. Am J Surg Pathol 2016;40(12):1702-12.

3. Kerr DA, Brcic I, Diaz-Perez JA, et al. Immunohistochemical characterization of giant cell tumor of bone treated with denosumab: support for osteoblastic differentiation. Am J Surg Pathol 2021;45(1):93-100.

4. Treffel M, Lardenois E, Larousserie F, et al. Denosumab-treated giant cell tumors of bone: a clinicopathologic analysis of 35 cases from the French group of bone pathology. Am J Surg Pathol 2020;44(1):1-10.

5. Jain SU, Khazaei S, Marchione DM, et al. Histone H3.3 G34 mutations promote aberrant PRC2 activity and drive tumor progression. Proc Natl Acad Sci U S A 2020;117(44):27354-64.

6. Fittall MW, Lyskjaer I, Ellery P, et al. Drivers underpinning the malignant transformation of giant cell tumour of bone. J Pathol 2020;252(4):433-40.

7. Amary F, Berisha F, Ye H, et al. H3F3A (Histone 3.3) G34W immunohistochemistry: a reliable marker defining benign and malignant giant cell tumor of bone. Am J Surg Pathol 2017;41(8):1059-68.

8. Behjati S, Tarpey PS, Presneau N, et al. Distinct H3F3A and H3F3B driver mutations define chondroblastoma and giant cell tumor of bone. Nat Genet Dec 2013;45(12):1479-82.

9. Presneau N, Baumhoer D, Behjati S, et al. Diagnostic value of H3F3A mutations in giant cell tumour of bone compared to osteoclast-rich mimics. J Pathol Clin Res 2015;1(2):113-23.

10. Lutsik P, Baude A, Mancarella D, et al. Globally altered epigenetic landscape and delayed osteogenic differentiation in H3.3-G34W-mutant giant cell tumor of bone. Nat Commun 2020;11(1): 5414.

11. Baumhoer D, Kovac M, Sperveslage J, et al. Activating mutations in the MAP-kinase pathway define non-ossifying fibroma of bone. J Pathol May 2019; 248(1):116-22.

12. Baumhoer D, Rogozhin DV. Non-ossifying fibroma. In: Board WCOTE, editor. WHO classification of tumours, soft tissue and bone tumours. 5th edition. France: IARC Press; 2020. p. 447-8.

13. Mallet JF, Rigault P, Padovani JP, et al. Non-ossifying fibroma in children: a surgical condition? Chir Pediatr 1980;21(3):179-89. Le fibrome non ossifiant chez l'enfant: une affection chirurgicale?

14. Schajowicz F, Ackerman LV, Sissons HA. Histologic typing of bone tumours. 1st edition WHO international histological classification of tumours. World Health Organization. Berlin: Springer; 1972.

15. Schajowicz F. Histologic typing of bone tumours. 2nd edition WHO international histological classification of tumours. World Health Organization. Berlin: Springer; 1993.

16. Nielsen GP, Kyriakos M. Non-ossifying fibroma/ benign fibrous histiocytoma of bone. In: Fletcher CDM, Bridge J, Hogendoorn PCW, et al, editors. WHO classification of tumours of soft tissue and bone. 4th edition. Lyon, France: IARC Press; 2013. p. 302-4.

17. Kuznetsov SA, Cherman N, Riminucci M, et al. Agedependent demise of GNAS-mutated skeletal stem cells and "normalization" of fibrous dysplasia of bone. J Bone Miner Res 2008;23(11):1731-40. 
18. Raubenheimer E, Jordan RC. Central giant cell granuloma. In: El-Naggar AK, Chan JKC, Grandis JR, et al, editors. WHO classification of head and neck tumours. Lyon, France: IARC Press; 2017. p. 256-7.

19. Raubenheimer E, Jordan RC. Peripheral giant cell granuloma. In: El-Naggar AK, Chan JKC, Grandis JR, et al, editors. WHO classification of head and neck tumours. Lyon, France: IARC Press; 2017. p. 257.

20. Gomes CC, Gayden T, Bajic A, et al. TRPV4 and KRAS and FGFR1 gain-of-function mutations drive giant cell lesions of the jaw. Nat Commun 2018; $9(1): 4572$.

21. Flanagan AM, Speight PM. Giant cell lesions of the craniofacial bones. Head Neck Pathol 2014;8(4): 445-53.

22. Pfeifer FM, Bridge JA, Neff JR, et al. Cytogenetic findings in aneurysmal bone cysts. Genes Chromosomes Cancer 1991;3(6):416-9.

23. Agaram NP, Bredella MA. Aneurysmal bone cyst. In: Board WCOTE, editor. WHO classification of tumours, soft tissue and bone tumours. 5th edition. Lyon, France: IARC Press; 2020. p. 437-9.

24. Forsyth R, Jundt G. Giant cell lesion of the small bones. In: Fletcher CDM, Bridge J, Hogendoorn PCW, et al, editors. WHO classification of tumours of soft tissue and bone. 4th edition. Lyon, France: IARC Press; 2013. p. 320.
25. Agaram NP, LeLoarer FV, Zhang L, et al. USP6 gene rearrangements occur preferentially in giant cell reparative granulomas of the hands and feet but not in gnathic location. Hum Pathol 2014;45(6): 1147-52.

26. Pizem J, Sekoranja D, Zupan A, et al. FUS-NFATC2 or EWSR1-NFATC2 fusions are present in a large proportion of simple bone cysts. Am J Surg Pathol 2020. https://doi.org/10.1097/PAS.000000000000 1584.

27. Flucke U, Shepard SJ, Bekers EM, et al. Fibroosseous pseudotumor of digits - Expanding the spectrum of clonal transient neoplasms harboring USP6 rearrangement. Ann Diagn Pathol 2018;35: 53-5.

28. Bekers EM, Eijkelenboom A, Grunberg K, et al. Myositis ossificans - Another condition with USP6 rearrangement, providing evidence of a relationship with nodular fasciitis and aneurysmal bone cyst. Ann Diagn Pathol 2018;34:56-9.

29. Paulson VA, Stojanov IA, Wasman JK, et al. Recurrent and novel USP6 fusions in cranial fasciitis identified by targeted RNA sequencing. Mod Pathol 2020;33(5):775-80.

30. Laaveri M, Heikinheimo K, Baumhoer D, et al. Periosteal fasciitis in a 7-year old girl: a diagnostic dilemma. Int J Oral Maxillofac Surg 2017;46(7): 883-5. 